\title{
Análise Comparativa da Intervenção Coronária Percutânea com Stents Farmacológicos versus Stents Não-Farmacológicos na Vigência de Infarto do Miocárdio com Supradesnivelamento do Segmento ST: Dados do Registro Brasileiro CENIC
}

\author{
Fernando Stucchi Devito1, José Antonio Marin-Neto², Luiz Alberto Mattos ${ }^{3}$
}

\section{RESUMO}

Fundamento: A intervenção coronária percutânea primária (ICPP) com stents farmacológicos apresenta resultados ainda incipientes e controversos na literatura. Objetivo: Comparar os resultados da ICPP com stents farmacológicos e não-farmacológicos no Brasil, no biênio 2006-2007. Método: De janeiro de 2006 a dezembro de 2007, todos os pacientes consecutivos acometidos de infarto agudo do miocárdio (IAM) com supradesnivelamento do segmento ST, tratados por meio da ICPP com stents farmacológicos ou não, devidamente relacionados no banco de dados da Central Nacional de Intervenções Cardiovasculares (CENIC) foram analisados quanto às características clínicas e angiográficas e aos resultados na fase hospitalar. Resultados: Foram analisados 4.876 pacientes tratados por meio da ICPP, e em 4.674 (95,9\%) dos quais foram utilizados stents não-farmacológicos e em $202(4,1 \%)$, stents farmacológicos. O sucesso do procedimento foi maior após os stents farmacológicos em relação aos não-farmacológicos $(97,5 \%$ vs. $93,5 \% ; p=0,02)$. Não houve diferenças entre os grupos quanto à trombose da endoprótese $(1,5 \%$ vs. $1,3 \% ; p=0,869)$ ou mesmo quanto à incidência geral de complicações $(1,0 \%$ vs. $2,8 \% ; p=0,119)$. A mortalidade tendeu a ser menor após stents farmacológicos $(2,0 \%$ vs. $4,4 \% ; p=0,098)$. Conclusão: No Brasil, a ICPP com stents farmacológicos apresenta baixas taxas de trombose e de mortalidade na fase hospitalar, comparáveis às taxas observadas com stents sem fármacos, em amostra populacional relativamente restrita de pacientes, nos quais

\section{SUMMARY}

Comparative Analysis of Percutaneous Coronary Intervention $(\mathrm{PCI})$ with Drug-eluting Stents versus Bare-Metal Stents in Acute ST-Segment Elevation Myocardial Infarction (STEMI): Data from the CENIC Registry

Background: Primary percutaneous coronary interventions (PPCl) with drug-eluting stents have incipient and still controversial results in the literature. Objective: To compare $\mathrm{PPCl}$ with drug-eluting and bare-metal stents in Brazil during the period 2006-2007. Method: All consecutive patients with $S T$-segment elevation myocardial infarction (STEMI) treated with $\mathrm{PPCl}$ with drug-eluting or bare-metal stents, reported to the database of the National Center for Cardiovascular Interventions (CENIC) from January 2006 to December 2007, were compared as to their clinical and angiographic characteristics and in-hospital results. Results: 4,876 patients treated with $\mathrm{PPCl}$ were analyzed; 4,674 (95.9\%) used bare-metal stents and 202 (4.1\%), drug-eluting stents. Procedural success rates were higher with drug-eluting stents $(97.5 \%$ vs. 93.5\%; $p=0.02)$. There were no differences in the rates of stent thrombosis $(1.5 \%$ vs. $1.3 \% ; p=0.869)$ or overall complications $(1.0 \%$ vs. $2.8 \% ; p=0.119)$. Mortality tended to be lower with drug-eluting stents $(2.0 \%$ vs. $4.4 \% ; p=0.098)$. Conclusion: In Brazil, PPCl with drug-eluting stents has a low rate of thrombosis and in-hospital mortality, compared to that observed for bare-metal stents in a relatively selected

\footnotetext{
1 Serviço de Cardiologia, Hospital São Domingos/Hospital Padre Albino - Catanduva, SP.

${ }^{2}$ Instituto Dante Pazzanese de Cardiologia - São Paulo, SP.

${ }^{3}$ Divisão de Cardiologia, Hospital das Clínicas da Faculdade de Medicina de Ribeirão Preto, USP - Ribeirão Preto, SP.

Correspondência: Fernando Stucchi Devito. Praça 9 de Julho, 176/111

- Centro - Catanduva, SP - CEP 15800-225

Recebido em: 30/8/2008 • Aceito em: 8/12/2008
} 
Devito FS, et al. Análise Comparativa da Intervenção Coronária Percutânea com Stents Farmacológicos versus Stents NãoFarmacológicos na Vigência de Infarto do Miocárdio com Supradesnivelamento do Segmento ST: Dados do Registro Brasileiro CENIC. Rev Bras Cardiol Invas. 2008;16(4):456-462.

a indicação desses stents foi certamente influenciada por fatores múltiplos e que afetaram os resultados comparativos.

DESCRITORES: Infarto do miocárdio. Angioplastia transluminal percutânea coronária. Angioplastia. Stents farmacológicos. Contenedores.
A reperfusão mecânica, por meio da intervenção coronária percutânea primária (ICPP), constitui o tratamento de escolha para pacientes com infarto agudo do miocárdio (IAM) com supradesnivelamento do segmento ST em até 12 horas do início do quadro, desde que possa ser iniciada em até 90 minutos do atendimento inicial'

Os stents coronários apresentam perfil de segurança satisfatório nesse cenário e, em comparação ao balão, possibilitam melhor evolução tardia, sobretudo pela menor necessidade de novos procedimentos de revascularização ${ }^{2-4}$. Em condições eletivas, vários estudos têm demonstrado que os stents farmacológicos inibem a hiperplasia intimal no local tratado e, conseqüentemente, reduzem a reestenose e a necessidade de um novo procedimento de revascularização em relação aos não-farmacológicos ${ }^{5-7}$. No entanto, os estudos que randomizaram os stents farmacológicos não incluíram número suficiente de pacientes com IAM e supradesnivelamento do segmento ST, de forma que a evidenciação de benefício dessa nova tecnologia nesse cenário clínico específico ainda é pouco consistente.

Além disso, estudos de pequeno porte, avaliando os stents farmacológicos no IAM com supradesnivelamento do segmento ST, apresentaram resultados controversos, com taxas de trombose e de morte acima do esperado para a nova tecnologia ${ }^{8-10}$. Também não se dispõem, ainda, de dados comprovando o potencial benefício dessas endopróteses, em comparação aos stents não-farmacológicos, em cenário menos controlado que o dos estudos randomizados, isto é, na prática clínica diária.

O Registro Brasileiro da Central Nacional de Intervenções Cardiovasculares (CENIC), agregando expressivo número de ICPP, é fonte de dados de consulta dinâmica e freqüente, emanados da prática comum, para a avaliação dos resultados dos stents farmacológicos nessa forma grave de apresentação clínica da doença arterial coronária (DAC), sem os vieses de seleção que costumeiramente ocorrem no âmbito dos estudos randômicos.

Assim, o objetivo deste estudo é analisar a evolução hospitalar dos pacientes com IAM com supradesnivelamento do segmento ST tratados por meio da ICPP com stents farmacológicos em comparação aos nãofarmacológicos no Registro Brasileiro da CENIC, no patient population, in which the referral for these stents was certainly influenced by multiple factors, which affected comparative results.

DESCRIPTORS: Myocardial infarction. Angioplasty, transluminal, percutaneous coronary. Angioplasty. Drug-eluting stents. Stents.

biênio 2006-2007, quando o uso daqueles dispositivos se intensificou ${ }^{11}$.

\section{MÉTODO}

A CENIC é um registro de intervenção percutânea cardiovascular da Sociedade Brasileira de Hemodinâmica e Cardiologia Intervencionista ( $\mathrm{SBHCl})$, existente há aproximadamente 18 anos, resultado da contribuição espontânea das informações dos procedimentos terapêuticos realizados pelos membros dessa Sociedade.

De janeiro de 2006 a dezembro de 2007, os dados de intervenções percutâneas realizadas foram enviados à CENIC exclusivamente por meio eletrônico, em ficha elaborada e aperfeiçoada a partir dos modelos preexistentes. Essa ficha contém informações referentes aos fatores de risco para DAC, quadro clínico dos pacientes, e dados referentes aos aspectos técnicos do procedimento realizado e à evolução na fase hospitalar.

Este estudo avaliou todos os pacientes consecutivos acometidos de IAM com supradesnivelamento do segmento ST, tratados por meio da ICPP com stents farmacológicos ou não, devidamente relacionados no banco de dados da CENIC, no biênio 2006-2007. As informações referentes às variáveis angiográficas qualitativas e quantitativas foram obtidas pela estimativa visual dos operadores. A extensão da DAC (vasos com $>50 \%$ de obstrução do lúmen arterial) foi quantificada em uni, biarterial, triarterial ou acometimento do tronco da artéria coronária esquerda (TCE). A função ventricular esquerda foi avaliada de acordo com a seguinte graduação: normal, discreto, moderado e grave déficit. Nesta análise, os vasos tratados foram agrupados em: artéria descendente anterior (DA), artéria circunflexa $(C X)$, artéria coronária direita (CD) ou outros (ramos ou enxertos venosos ou arteriais). O tipo de stent utilizado, assim como o diâmetro e a extensão da endoprótese implantada, também foram reportados.

As definições utilizadas são as já consagradas e utilizadas nas Diretrizes de Intervenção Coronária Percutânea e Métodos Adjuntos Diagnósticos em Cardiologia Intervencionista da $\mathrm{SBHCl}$, recentemente publicadas $^{12}$. O sucesso do procedimento foi definido angiograficamente (redução da estenose-alvo para grau de estenose $<30 \%$, com restabelecimento do fluxo 
Devito FS, et al. Análise Comparativa da Intervenção Coronária Percutânea com Stents Farmacológicos versus Stents NãoFarmacológicos na Vigência de Infarto do Miocárdio com Supradesnivelamento do Segmento ST: Dados do Registro Brasileiro CENIC. Rev Bras Cardiol Invas. 2008;16(4):456-462.

anterógrado grau-3, pela classificação de $\mathrm{TIMI}^{13}$ ) e ausência de complicações clínicas graves (morte, reinfarto, e cirurgia em fase hospitalar). O reinfarto foi caracterizado pela presença de alterações eletrocardiográficas (novo supradesnivelamento do segmento ST ou novas "Q") e/ou evidência angiográfica de oclusão do vasoalvo. A trombose do stent compreendeu a ocorrência de oclusão aguda e subaguda do vaso, comprovada angiograficamente. Foram consideradas para análise a mortalidade geral e a mortalidade de causa cardíaca. Essas definições foram julgadas nos centros participantes desse Registro, por cardiologistas intervencionistas devidamente habilitados, que contribuem com a CENIC.

A análise estatística foi realizada com o programa de software SPSS versão 15.0. As variáveis contínuas foram expressas em médias e desvios padrão e as categóricas, em freqüências absolutas e porcentagens. Na comparação entre os grupos (stents convencionais e farmacológicos), foram utilizados os testes $t$ de Student, para variáveis quantitativas, e qui-quadrado, para variáveis categóricas. Foram considerados significativos os valores de $p<0,05$.

\section{RESULTADOS}

De janeiro de 2006 a dezembro de 2007, foram analisados 4.876 pacientes consecutivos que apresentaram IAM com supradesnivelamento do segmento ST e que foram tratados por meio da ICPP com stents, sendo utilizados stents farmacológicos Taxus $^{\circledR}$ em $51 \%$, Cypher $^{\circledR}$ em $22 \%$, Endeavor ${ }^{\circledR}$ em $15 \%$ e outros em $12 \%$ dos casos) em $202(4,1 \%)$ pacientes e stents nãofarmacológicos em 4.674 (95,9\%).

A média de idade $(61,8$ anos $\pm 13,8$ anos versus $61,9$ anos $\pm 12,8$ anos; $p=0,920)$ e a proporção de mulheres $(31,6 \%$ vs. $27,2 \%)$ foram semelhantes nos dois grupos de tratamento. Os pacientes tratados com stents farmacológicos apresentaram maior freqüência de diabetes melito $(31,0 \%$ vs. $21,3 \% ; p=0,002)$, dislipidemia $(67,4 \%$ vs. $55,8 \% ; p=0,002)$ e infarto prévio $(15,5 \%$ vs. $10,3 \% ; p=0,024)$, apesar da menor prevalência de tabagismo $(31,0 \%$ vs. $41,5 \% ; p=0,004)$ e hipertensão arterial sistêmica $(68,4 \%$ vs. $77,6 \%$; $\mathrm{p}=0,004)$ nesse grupo de pacientes. Com relação aos antecedentes de revascularização, os pacientes com stents farmacológicos apresentaram mais intervenções coronárias percutâneas (ICPs) prévias (19,3\% vs. 7,9\%; $p<0,001)$, realizadas em lesões reestenóticas $(6,4 \%$ vs. $1,6 \% ; p<0,001)$. As características clínicas estão descritas na Tabela 1.

Quanto à avaliação da gravidade do quadro clínico do infarto no momento da intervenção, segundo a classificação de Killip-Kimball, não houve diferenças significativas entre os dois grupos de tratamento em quaisquer estágios da classificação, de I a IV ( $p=0,178)$.

Não houve diferenças entre os grupos quanto à presença ou não de circulação colateral na cinecoro- nariografia pré-intervenção $(p=0,859)$, nem mesmo quanto ao tempo de retardo do diagnóstico à realização da ICP (117 minutos \pm 132 minutos vs. 134 minutos \pm 160 minutos; $p=0,077$ ), mas o grupo dos stents farmacológicos já se apresentava inicialmente com maior prevalência de fluxo coronário TIMI-1, enquanto a maioria $(63,8 \%)$ dos pacientes tratados com stents não-farmacológicos se apresentou com fluxo TIMI-0 antes do procedimento (Figura 1).

Os pacientes com stents farmacológicos apresentaram lesões mais longas (41,8\% versus $27,9 \%$; $\mathrm{p}<0,001)$ e as endopróteses utilizadas nesse grupo foram de maior extensão (média de extensão do stent de 22,5 mm vs. 19,9 mm; $p<0,001$ ) e de menor diâmetro (média do diâmetro de 2,94 mm vs. 3,11 mm; $p<0,001)$, em comparação aos stents não-farmacológicos. Não houve diferenças quanto à extensão da DAC, mas com menor freqüência os stents farmacológicos foram implantados na $C X(p<0,001)$. As características angiográficas encontram-se na Tabela 2.

Com relação à farmacologia adjunta, o emprego de inibidor da glicoproteína Ilb/IIla (GPIIb/IIla) foi semelhante nos dois grupos de tratamento $(23,3 \%$ vs. $25,7 \% ; p=0,440)$. A maioria dos pacientes (>98\%) recebeu ácido acetilsalicílico, mas o clopidogrel foi prescrito, na fase hospitalar, com maior freqüência aos pacientes com stents farmacológicos $(98,0 \%$ vs. 87,2\%; $\mathrm{p}<0,001)$.

O sucesso do procedimento ocorreu com maior freqüência após os stents farmacológicos em relação aos não-farmacológicos (97,5\% versus $93,5 \% ; p=0,021)$. Não houve diferenças entre os grupos quanto à incidência de reinfarto e/ou de trombose da endoprótese $(1,5 \%$ vs. $1,3 \% ; p=0,869)$. Dos três $(1,5 \%)$ pacientes que apresentaram trombose após stents farmacológicos, foi realizada nova ICP em dois deles. Dos 63 (1,3\%) pacientes com trombose após stents não-farmacológicos, $37(0,8 \%)$ foram tratados por meio de nova ICP e $6(0,1 \%)$ foram encaminhados para cirurgia de emergência. Quanto à incidência de outras complicações, entre os 202 pacientes tratados com stents farmacológicos houve ocorrência apenas de um acidente vascular cerebral (AVC) e de um sangramento leve. Entre os 4.674 pacientes com stents não-farmacológicos, $16(0,3 \%)$ apresentaram AVC, $50(1,1 \%)$ apresentaram sangramentos graves e $66(1,4 \%)$, sangramentos leves. Na análise conjunta dessas complicações, não houve diferença entre os dois grupos de tratamento $(1,0 \%$ vs. $2,8 \% ; p=0,119)$. A mortalidade tendeu a ser menor após stents farmacológicos, mas sem significância estatística (2,0\% vs. 4,4\%; $p=0,098)$. Os resultados encontram-se na Tabela 3 .

\section{DISCUSSÃO}

Uma primeira constatação deste estudo é que, mesmo nesse biênio mais recente, a penetração dos 
Devito FS, et al. Análise Comparativa da Intervenção Coronária Percutânea com Stents Farmacológicos versus Stents NãoFarmacológicos na Vigência de Infarto do Miocárdio com Supradesnivelamento do Segmento ST: Dados do Registro Brasileiro CENIC. Rev Bras Cardiol Invas. 2008;16(4):456-462.

TABELA 1

Variáveis clínicas dos pacientes tratados com intervenção coronária percutânea primária com stents farmacológicos versus stents não-farmacológicos no Registro CENIC (biênio 2006-2007)

\begin{tabular}{lccc}
\hline Variáveis & Stents farmacológicos $(\mathbf{n}=\mathbf{2 0 2})$ & Stents não-farmacológicos $(\mathbf{n}=\mathbf{4 . 6 7 4 )}$ & $\mathbf{p}$ \\
\hline Gênero feminino (\%) & 27,2 & 31,6 & 0,186 \\
Idade em anos, média (DP) & $61,9(12,8)$ & $61,8(13,8)$ & 0,920 \\
Fatores de risco para DAC & & & 0,004 \\
$\quad$ Hipertensão arterial sistêmica & 68,4 & 77,6 & 0,002 \\
$\quad$ Diabetes melito & 31,0 & 21,3 & 0,643 \\
$\quad$ Insulino-dependente & 12,2 & 14,3 & 0,004 \\
Tabagismo & 31,0 & 41,5 & 0,002 \\
$\quad$ Dislipidemia & 67,4 & 55,8 & 0,024 \\
Infarto prévio & 15,5 & 10,3 & 0,271 \\
História familiar de DAC & 32,1 & 28,4 & 0,305 \\
Cirurgia de RM prévia & 5,0 & 3,6 & $<0,001$ \\
ICP prévia & 19,3 & 7,9 & \\
\hline
\end{tabular}

CENIC = Central Nacional de Intervenções Cardiovasculares; DAC = doença arterial coronária; DP = desvio padrão; ICP = intervenção coronária percutânea; $\mathrm{n}$ = número de pacientes; $\mathrm{RM}$ = revascularização miocárdica.

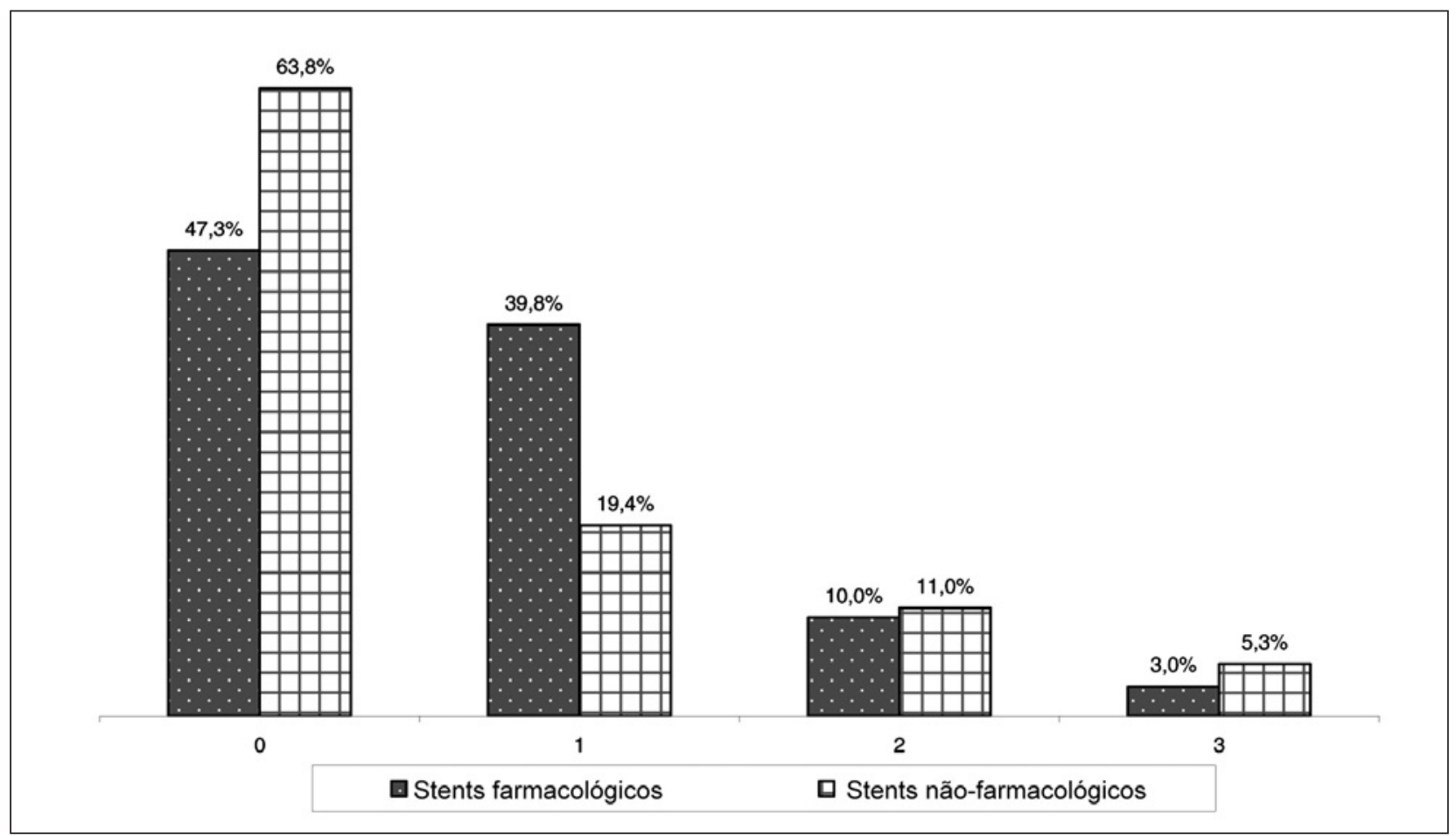

Figura 1 - Prevalência dos graus de fluxo coronário pré-intervenção coronária percutânea (classificação de TIMI) em pacientes tratados com stents farmacológicos versus não-farmacológicos no Registro CENIC (biênio 2006-2007). p 0,001.

stents farmacológicos na ICCP para pacientes com IAM e supradesnivelamento do segmento ST $(4,1 \%)$ é ainda pequena em comparação à observada nos procedimentos eletivos, que já em 2004 correspondia a $14 \%$ das ICPs no Brasil ${ }^{11}$. Outras séries também recentes na literatura corroboram esses dados, mesmo em países nos quais a questão do custo não é fator limitante da escolha do instrumental na ICPP ${ }^{14}$.

Uma das possíveis razões para esse fato poderia ser a consideração de que a existência de menor carga de placa de ateroma no IAM - placas mais rasas se 
Devito FS, et al. Análise Comparativa da Intervenção Coronária Percutânea com Stents Farmacológicos versus Stents NãoFarmacológicos na Vigência de Infarto do Miocárdio com Supradesnivelamento do Segmento ST: Dados do Registro Brasileiro CENIC. Rev Bras Cardiol Invas. 2008;16(4):456-462.

TABELA 2

Variáveis angiográficas dos pacientes tratados com intervenção coronária percutânea primária com stents farmacológicos versus stents não-farmacológicos no Registro CENIC (biênio 2006-2007)

\begin{tabular}{|c|c|c|c|}
\hline Variáveis & $\begin{array}{l}\text { Stents farmacológicos } \\
\qquad(\mathrm{n}=202)\end{array}$ & $\begin{array}{l}\text { Stents não-farmacológicos } \\
(\mathrm{n}=4.674)\end{array}$ & $\mathbf{p}$ \\
\hline Disfunção contrátil VE moderada e/ou grave (\%) & 31,7 & 30,3 & 0,674 \\
\hline Extensão da DAC (\%) & & & 0,626 \\
\hline Uniarterial & 40,5 & 43,1 & \\
\hline Biarterial & 33,3 & 33,7 & \\
\hline Triarterial & 25,5 & 22,3 & \\
\hline Lesão TCE & 0,7 & 1,0 & \\
\hline Vaso tratado $(\%)$ & & & $<0,001$ \\
\hline DA & 57,4 & 42,4 & \\
\hline $\mathrm{CX}$ & 24,3 & 44,4 & \\
\hline $\mathrm{CD}$ & 15,8 & 12,1 & \\
\hline Outros & 2,5 & 1,1 & \\
\hline Presença de circulação colateral (\%) & & & 0,859 \\
\hline Grau I & 54,3 & 56,1 & \\
\hline Grau II & 37,0 & 32,6 & \\
\hline Grau III & 6,5 & 9,6 & \\
\hline Grau IV & 2,2 & 1,7 & \\
\hline \multicolumn{4}{|l|}{ Características da lesão tratada (\%) } \\
\hline Calcificação & 23,4 & 20,8 & 0,381 \\
\hline Extensão > 20 mm & 41,8 & 27,9 & $<0,001$ \\
\hline Tipo da lesão B2+C* & 83,7 & 84,9 & 0,657 \\
\hline Ramos secundários envolvidos & 32,3 & 26,7 & 0,080 \\
\hline Diâmetro do stent em mm, média (DP) & $2,94(0,39)$ & $3,11(0,47)$ & $<0,001$ \\
\hline Extensão do stent em mm, média (DP) & $22,45(6,80)$ & $19,85(6,30)$ & $<0,001$ \\
\hline
\end{tabular}

instabilizando e rompendo - pode resultar em menor tendência de reestenose, predispondo, assim, a diminuir o potencial de benefício de estratégia antireestenótica, representada pelos stents farmacológicos nessa forma de apresentação clínica da DAC. Mas, muito provavelmente, a ausência de dados mais robustos que comprovem o benefício do stent farmacológico na ICPP a longo prazo e o número ainda modesto de pacientes em estudos dedicados à questão constituem razões adicionais para esse fato.

O estudo TYPHOON, que randomizou número mais expressivo de pacientes, ou seja, 712 pacientes em 48 centros, apesar de ter demonstrado a superioridade do stent com sirolimus na redução da necessidade de reintervenção sobre o vaso-alvo em um ano (7,3\% vs. 14,3\%; $p=0,004)$, não demonstrou diferenças quanto a mortalidade, reinfarto ou trombose do stent $^{15}$. Também no estudo PASSION, com 619 pacientes randomizados, o uso do stent com paclitaxel não resultou em menor incidência dos eventos com- binados morte, reinfarto ou nova revascularização $(8,8 \%$ vs. $12,8 \% ; p=0,09)^{16}$.

A segunda conclusão a ressaltar neste estudo é que, com poucas exceções, ao se analisar as variáveis clínicas e angiográficas em ambos os grupos, evidencia-se seleção dos pacientes de maior complexidade, incluindo maior prevalência de diabetes melito, menor diâmetro dos vasos e maior extensão das lesões, fatores diretamente relacionados à ocorrência de reestenose, e da maior necessidade de um novo procedimento na evolução.

Apesar da maior complexidade dos pacientes e das lesões observadas no grupo tratado com stents farmacológicos, a taxa de sucesso imediato foi mais elevada, comparativamente à observada após implante dos stents convencionais $(97,5 \%$ vs. 93,5\%; $p=0,02)$. Como a decisão pelo implante de um stent farmacológico em relação a um não-farmacológico leva em consideração principalmente a perspectiva de manutenção tardia do resultado angiográfico obtido e não 
Devito FS, et al. Análise Comparativa da Intervenção Coronária Percutânea com Stents Farmacológicos versus Stents NãoFarmacológicos na Vigência de Infarto do Miocárdio com Supradesnivelamento do Segmento ST: Dados do Registro Brasileiro CENIC. Rev Bras Cardiol Invas. 2008;16(4):456-462.

TABELA 3

Resultados da intervenção coronária percutânea primária com stents farmacológicos versus stents não-farmacológicos no Registro CENIC (biênio 2006-2007)

\begin{tabular}{lccc}
\hline Variáveis & Stents farmacológicos $(\mathbf{n}=\mathbf{2 0 2})$ & Stents não-farmacológicos $(\mathbf{n}=\mathbf{4 . 6 7 4})$ & $\mathbf{p}$ \\
\hline Sucesso do procedimento (\%) & 97,5 & 93,5 & 0,021 \\
Óbito (\%) & 2,0 & 4,4 & 0,098 \\
Óbito cardíaco (\%) & 2,0 & 3,9 & 0,164 \\
Reinfarto (\%) & 1,5 & 1,3 & 0,869 \\
ICP de urgência (\%) & 1,0 & 0,8 & 0,676 \\
Cirurgia de emergência (\%) & 0 & 0,1 & $>0,999$ \\
Trombose do stent (\%) & 1,5 & 1,3 & 0,869 \\
AVC (\%) & 0,5 & 0,3 & 0,513 \\
Insuficiência renal aguda (\%) & 0,5 & 0,6 & 0,851 \\
\hline
\end{tabular}

AVC = acidente vascular cerebral; CENIC = Central Nacional de Intervenções Cardiovasculares; ICP = intervenção coronária percutânea; $\mathrm{n}$ = número de pacientes.

a obtenção de qualquer ganho imediato, clínico ou angiográfico, esses resultados são de difícil interpretação. É bastante provável que a maior freqüência de fluxo TIMI-1, pré-intervenção, nos pacientes tratados com stents farmacológicos, tenha contribuído para o maior sucesso do procedimento, pois quanto mais precoce o restabelecimento, mesmo parcial, do fluxo coronário maior é o benefício da reperfusão. Também o uso menos freqüente de clopidogrel no grupo de pacientes tratados com os stents não-farmacológicos pode ter acrescentado maior risco a esse grupo, ainda na fase hospitalar. Deve-se assinalar que o uso de inibidores GPIIb/IIla, em ambos os grupos, foi pelo menos duas vezes superior ao habitualmente registrado em pacientes tratados eletivamente com ICP no cenário nacional ( $<10 \%$ ), revelando, dessa maneira, nicho de utilização clínica desses fármacos no Brasil.

A questão mais controversa a influenciar a utilização dos stents farmacológicos no IAM com supradesnivelamento do segmento ST é a da segurança. A trombose do stent, em nosso estudo, ocorreu em taxas aceitáveis $(<1,5 \%)$, ainda mais se levarmos em consideração o contexto clínico da intervenção. Estudos recentes relatam taxas hospitalares de trombose da endoprótese ao redor de $2 \%$, o que ratifica o perfil de segurança dos stents, mesmo quando implantados em uma placa instável e na presença de trombo ${ }^{17,18}$. No recente estudo HORIZONS-AMI, com 3.602 pacientes com IAM com supradesnivelamento do segmento ST, randomizados para stents farmacológicos versus nãofarmacológicos, não houve diferença quanto à incidência de trombose do stent $(3,1 \%$ vs. $3,4 \% ; p=0,72)$ ou quanto à mortalidade $(3,5 \%$ vs. 3,5\%; $p=0,92)$ em um ano de evolução. Já a revascularização da lesão-alvo foi menor após o stent farmacológico (4,5\% vs. 7,5\%; $\mathrm{p}=0,002)^{19}$.
No Registro GRACE, pacientes com IAM apresentaram, após stents farmacológicos, mortalidade duas vezes maior que a observada após stents sem fármacos, na evolução de dois anos ${ }^{20}$. Em contraste, nosso estudo evidenciou mortalidade em geral muito baixa, mesmo em se tratando de pacientes de alto risco e pertencentes a um registro de dimensão nacional: a mortalidade hospitalar após stents farmacológicos foi de 2,2\%, inferior à observada após stents não-farmacológicos, apesar de a diferença não ter alcançado significância estatística. Nossos resultados estão de acordo com os relatados em outro grande registro, com 7.217 pacientes apresentando IAM e sendo tratados com ICPP: após dois anos de evolução, verificou-se menor mortalidade nos pacientes com IAM com supradesnivelamento do segmento ST tratados com stents farmacológicos em relação aos não-farmacológicos $(8,5 \% \text { vs. } 11,6 \% ; p=0,008)^{21}$. As curvas de mortalidade relativas aos dois grupos de stents se separaram precocemente nos primeiros dias após o infarto e se mantiveram assim pelo tempo de evolução do estudo. No contexto atual, isso ainda requer confirmação com estudos randomizados que analisem adequadamente o mérito da questão, mas deve-se enfatizar que os resultados de nossa análise, no âmbito do registro CENIC, tendem a corroborar os dados desse estudo.

A primeira limitação do nosso estudo refere-se à própria natureza da análise, retrospectivamente idealizada, e com dados não adjudicados. Um segundo ponto de limitação é o da grande diferença numérica entre os dois grupos. Mesmo assim, o grupo de pacientes tratados com stents farmacológicos no IAM com supradesnivelamento do segmento ST demonstra resultados expressivos e verificados em outros estudos similares que refletem a prática no mundo real das intervenções. 
Devito FS, et al. Análise Comparativa da Intervenção Coronária Percutânea com Stents Farmacológicos versus Stents NãoFarmacológicos na Vigência de Infarto do Miocárdio com Supradesnivelamento do Segmento ST: Dados do Registro Brasileiro CENIC. Rev Bras Cardiol Invas. 2008;16(4):456-462.

\section{CONCLUSÕES}

No Brasil, a utilização de stents farmacológicos na ICPP é baixa, em comparação à observada em procedimentos eletivos de revascularização percutânea. Apesar da maior complexidade clínica e angiográfica, indicando certo viés de seleção dos operadores, no sentido de usar esse recurso em mais difíceis condições de trabalho, os pacientes com IAM com supradesnivelamento do segmento ST, tratados por meio da ICPP com stents farmacológicos, apresentam baixas taxas de trombose e de mortalidade na fase hospitalar, comparáveis às observadas com stents não-farmacológicos.

\section{REFERÊNCIAS BIBLIOGRÁFICAS}

1. Keeley EC, Boura JA, Grines CL. Primary angioplasty versus intravenous thrombolytic therapy for acute myocardial infarction: a quantitative review of 23 randomised trials. Lancet. 2003;361(9351):13-20.

2. Zhu MM, Feit A, Chadow H, Alam M, Kwan T, Clark LT. Primary stent implantation compared with primary balloon angioplasty for acute myocardial infarction: a meta-analysis of randomized clinical trials. Am J Cardiol. 2001;88(3):297-301.

3. Stone GW, Grines CL, Cox DA, Garcia E, Tcheng JE, Griffin JJ, et al. Comparison of angioplasty with stenting, with or without abciximab, in acute myocardial infarction. $\mathrm{N}$ Engl J Med. 2002;346(13):957-66.

4. De Luca G, Suryapranata H, Stone GW, Antoniucci D, Biondi-Zoccai G, Kastrati A, et al. Coronary stenting versus balloon angioplasty for acute myocardial infarction: a metaregression analysis of randomized trials. Int J Cardiol. 2008; 126(1):37-44.

5. Sousa JE, Costa MA, Abizaid A, Abizaid AS, Feres F, Pinto IM, et al. Lack of neointimal proliferation after implantation of sirolimus-coated stents in human coronary arteries: a quantitative coronary angiography and three-dimensional intravascular ultrasound study. Circulation. 2001;103(2):192-5.

6. Sousa JE, Costa MA, Abizaid A, Feres F, Seixas AC, Tanajura $L F$, et al. Four-year angiographic and intravascular ultrasound follow-up of patients treated with sirolimus eluting stents. Circulation. 2005;111(18):2326-9.

7. Daemen J, Serruys PW. Drug-eluting stent update 2007: part II. Unsettled issues. Circulation. 2007;116(8):961-8.

8. Cook $S$, Wenaweser $P$, Togni $M$, Billinger $M$, Morger $C$, Seiler C, et al. Incomplete stent apposition and very late stent thrombosis after drug-eluting stent implantation. Circulation. 2007;115(18):2426-34.

9. Daemen J, Tanimoto S, García-García HM, Kukreja N, van de Sande M, Sianos G, et al. Comparison of three-year clinical outcome of sirolimus- and paclitaxel-eluting stents versus bare metal stents in patients with ST-segment eleva- tion myocardial infarction (from the RESEARCH and T-SEARCH Registries). Am J Cardiol. 2007;99(8):1027-32.

10. Sianos G, Papafaklis MI, Daemen J, Vaina S, van Mieghem CA, van Domburg RT, et al. Angiographic stent thrombosis after routine use of drug-eluting stents in ST-segment elevation myocardial infarction: the importance of thrombus burden. J Am Coll Cardiol. 2007;50(7):573-83.

11. Cardoso CO, Quadros AS, Mattos LA, Gottschall CA, SarmentoLeite R, Marin-Neto JA. Perfil de uso dos stents farmacológicos no Brasil: dados da Central Nacional de Intervenções Cardiovasculares (CENIC). Arq Bras Cardiol. 2007;89(6):356-61.

12. Mattos LAP, Lemos Neto PA, Rassi Jr A, Marin-Neto JA, Sousa AGMR, Devito FS, et al. Diretrizes de Intervenção Coronária Percutânea e Métodos Adjuntos Diagnósticos em Cardiologia Intervencionista da $\mathrm{SBHCl}$. Rev Bras Cardiol Invas. 2008;16 Supl 2:9-88.

13. TIMI Study Group. The Thrombolysis in Myocardial Infarction (TIMI) trial. Phase I findings. N Engl J Med. 1985;312(14):932-6.

14. Percoco G, Manari A, Guastaroba P, Campo G, Guiducci $\mathrm{V}$, Aurier E, et al. Safety and long-term efficacy of sirolimus eluting stent in ST-elevation acute myocardial infarction: the REAL (Registro REgionale AngiopLastiche Emilia-Romagna) registry. Cardiovasc Drugs Ther. 2006;20(1):63-8.

15. Spaulding C, Henry P, Teiger E, Beatt K, Bramucci E, Carrié D, et al. Sirolimus-eluting versus uncoated stents in acute myocardial infarction. N Engl J Med. 2006;355(11):1093-104.

16. Laarman GJ, Suttorp MJ, Dirksen MT, van Heerebeek L, Kiemeneij F, Slagboom T, et al. Paclitaxel-eluting versus uncoated stents in primary percutaneous coronary intervention. $\mathrm{N}$ Engl J Med. 2006;355(11):1105-13.

17. Kastrati A, Dibra A, Spaulding C, Laarman GJ, Menichelli M, Valgimigli $M$, et al. Meta-analysis of randomized trials on drug-eluting stents vs. bare-metal stents in patients with acute myocardial infarction. Eur Heart J. 2007;28(22):2706-13.

18. De Luca G, Stone GW, Suryapranata H, Laarman G), Menichelli M, Kaiser C, et al. Efficacy and safety of drug-eluting stents in ST-segment elevation myocardial infarction: A meta-analysis of randomized trials. Int J Cardiol. 2008. [Epub ahead of print].

19. Stone GW, for the HORIZONS-AMI Investigators. A prospective, randomized comparison of paclitaxel eluting Taxus stents vs. bare metal stents during primary angioplasty in acute myocardial infarction - one year results. In: $20^{\text {th }}$ Annual Transcatheter Cardiovascular Therapeutics (TCT) 2008; Washington. Disponível em: http://www.tctmd.com/txshow. aspx?tid=2640\&id=70522\&trid=2. Acesso em: 24/11/2008.

20. Steg PG. Increased all-cause mortality at 2-year follow-up after $\mathrm{PCl}$ with drug-eluting stents versus bare-metal stents in acute coronary syndromes: the GRACE Registry. In: European Society of Cardiology World Congress. Vienna, 2007.

21. Mauri L, Silbaugh TS, Garg P, Wolf RE, Zelevinsky K, Lovett A, et al. Drug-eluting or bare-metal stents for acute myocardial infarction. N Engl J Med. 2008;359(13):1330-42. 